\title{
Simulations of polycrystalline growth in $2+1$ dimensions
}

\author{
J. M. Thijssen \\ University of Nijmegen, Toernooiveld, 6525 ED Nijmegen, The Netherlands
}

(Received 9 June 1994)

\begin{abstract}
Simulations of the Van der Drift model for polycrystalline growth are described. These simulations have been carried out for conic and cubic crystals and the exponent $p$ describing the growth of the linear monocrystalline domain size has been determined. For both crystal types $p=0.4$ is found, in accordance with previously obtained mean-field results. Moreover, the angular distribution of surviving crystals as a function of time matches the mean-field result very well.
\end{abstract}

\section{INTRODUCTION}

Polycrystalline films have received growing interest in the last years. Especially diamond with its extreme hardness and optical properties is a technologically interesting material for such films and many experiments are carried out using this material. ${ }^{1}$ Computer simulations can be a valuable support for experimental work in this field. ${ }^{2}$ Such simulations are usually performed for the Van der Drift model..$^{3,4,2,5}$ In this model, the growth of a crystal is normal to its facets, and for one type of facet, the growth rate is independent of the orientation of the facet with respect to the film. Normally, the growth rates of all facet types are taken to be equal. Initially, a collection of such crystals is placed on a flat ground plane $(2+1$ dimensions) or line $(1+1)$ with random orientation. The evolution of the model is then uniquely determined in $(1+1)$ dimensions but in $(2+1)$ dimensions extra information has to be given to describe the evolution from a point where more than three facets meet (see below).

In the course of time, crystals will disappear from the surface. The crystals whose tops are pointing more or less normal to the surface will persist longer at the surface than those pointing in less favorable directions. The model is a competitive growth model, other examples of which are the so-called "grass models." 6

The validity of the Van der Drift model for describing experimental situations can be tested by comparing computer simulation results with $\mathrm{x}$-ray texture analysis data. $^{2}$ To our knowledge, only for the $(1+1)$ dimensional version simulations have been carried out up to this moment, which can only allow for a qualitative comparison, which is promising. ${ }^{2}$

Another interesting property which can be determined in simulations is the number $N$ of crystals surviving at the surface as a function of time. This number is related to the linear domain size, or correlation length $\xi$ by $N \sim$ $\xi^{-d}$ for the $(d+1)$ dimensional model. Because of the absence of an intrinsic scale in the problem, it is clear that this number scales with time as a power law:

$$
\xi \sim t^{p}
$$

Dammers and Radelaar ${ }^{4}$ have determined the exponent $p$ for the $(1+1)$ dimensional model numerically. They found $p=0.5$, a result which was reproduced by a meanfield analysis. ${ }^{7}$ This analysis can be extended to three dimensions, and for this case, an exponent $p=0.4$ is predicted. ${ }^{8}$ Kolmogorov also analyzed the same problem as early as $1949 .{ }^{9}$ He found $p=0.5$ for $(1+1)$ dimensions by scaling arguments and conjectured the same value $p=$ 0.5 for $(2+1)$ dimensions.

In the mean-field analysis correlations arising during the growth are neglected: the probability for the survival of a crystal with a specific orientation is taken to be independent of its actual environment. In practice, however, the existence of "fit" crystals (i.e., having tops pointing in a direction more or less normal to the surface) will decrease the probability for less fit ones to survive in their neighborhood. Another approximation made in the mean-field calculation resides in the fact that survival of a crystal is defined in terms of its top only: as soon as a crystal top disappears, the surviving trunk is neglected. Numerical results for the $(1+1)$ dimensional case, however, showed that both approximations do not affect the value of the exponent $p$. It is not a priori clear that this be the case also in $(2+1)$ dimensions. Therefore, the validity of the mean-field exponent in the Van der Drift model should be verified by computer simulations. Such simulations have been performed and are presented in this paper.

Another type of films are the amorphous ones. These have been studied both theoretically and experimentally in the literature to great extent. ${ }^{10}$ Generally, self-affine fractal structures arise in such films, leading to similarity scaling or Hurst exponents that can be related to correlation-length exponents. Most of these structures are believed to be described adequately by the Kardar Parisi Zhang (KPZ) equation. ${ }^{11}$ In fact, growth normal to the surface of our model is also included in this equation. If one neglects the Langevin noise term and the diffusion in the KPZ equation, the resulting equation,

$$
\frac{d h}{d t}(t)=\sqrt{1-\left(\frac{d h}{d x}\right)^{2}}
$$

describes normal growth. However, the KPZ equation is not solvable in a strict sense since it does not specify the evolution of an upward-pointing singularity, like a crystal 


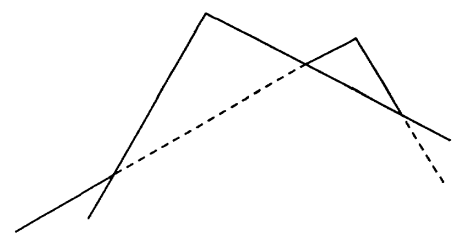

FIG. 1. Two cones in $(1+1)$ dimensions. The left one has disappeared in the right one, and is not allowed to pop up at the right flank of the latter. Therefore, the situation depicted here will not occur.

top. ${ }^{7}$ If such a top is taken to evolve into a spherical structure, one gets the Huygens growth model, studied by Tang et $a l .{ }^{12}$ In the Van der Drift model, the top remains sharp.

\section{THE THREE-DIMENSIONAL VAN DER DRIFT MODEL}

The Van der Drift model is a local model: borders between the visible parts of different crystals will shift but it is impossible for one or more crystal facets to emerge suddenly between or inside one or more others. In $(1+1)$ dimensions this prohibits for example events of the type depicted in Fig. 1 to occur. The evolving structure, therefore, cannot be viewed as the envelope of the original crystals if they were to grow without restriction: the entire evolution from the moment that the crystals touch each other has to be considered to determine the structure for arbitrary times.

When growing three-dimensional (3D) crystals, the prescription of normal growth and conserving the topology of the tops and edges is not sufficient to specify the evolution. An example is shown in Fig. 2. Four edges merge in one point, and two different evolutions are possible. The same problem occurs for a situation as represented in Fig. 3.

For such ambiguous events, we will stick to the prescription that of all possibilities the one yielding the greatest height for every position on the ground plane near the event is chosen. It should be noted that for the case shown in Fig. 3, this approach is not realistic

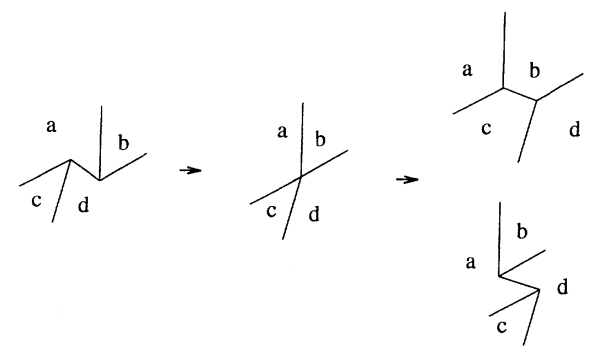

FIG. 2. Five edges separating four facets $a-d$. The two vertices in the left picture approach each other. They merge in the middle picture. Now two different evolutions are possible, both shown in the upper and lower picture on the right.
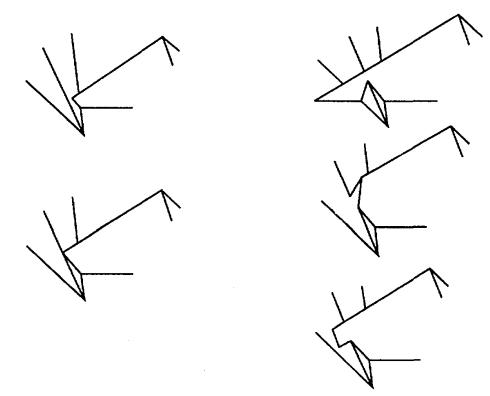

FIG. 3. A crystal reaching over another one. The left hand side shows the evolution up to the moment that the right crystal reaches over the left one. On the right hand side, three possible further evolutions are shown. The picture at the top shows the convention adopted in our simulation. The least realistic one is seen in the middle: the face of the left crystal is continued to cut off the right crystal. The lower picture shows the most realistic approach: a genuine overhang with facets belonging to the right crystal.

since, if one crystal reaches over another one, suddenly a complete segment belonging to this crystal arises at the "overhang" side. The realistic approach, which consists of letting an overhang occur with facets determined by the overhanging crystal, is hard to treat in a simulation, since one can no longer specify the system by its twodimensional projection on the ground plane in that case. It is believed that this choice for the evolution does not affect the exponent $p$, which is related to the behavior of the tops, which are not directly involved in such events.

Instead of treating faceted crystals, one can consider cones, with uniform growth rate normal to the tangent plane on the surface. Cones can be viewed as a limiting case of multifaceted crystals. For cones, the problems of overhangs ceases to exist. The analog of the situation depicted in Fig. 3 cannot occur here. To see this, suppose one cone is just at the point of reaching over another one, as in Fig. 4. As the growth velocity is normal to the tangent planes of both cones, in particular, at the "reachover" point, it is clear that their respective topology will not change and has been the same for earlier times.

\section{THE SIMULATION AND RESULTS}

I have performed simulations for both cubic and conic crystals. The "cubic" crystals consist only of three per-

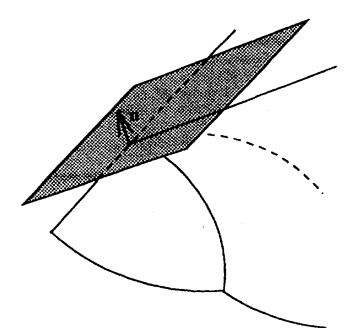

FIG. 4. Two cones of which the right one is at the point of reaching over the left one. The shaded plane is tangent to both cones at the point $P$. Therefore, this geometry is stationary. 
pendicular facets, to avoid overhangs. In these simulations, the crystals are put on a regular grid on the ground plane. Their growth axes are randomly distributed. The polar angle $\theta$ is uniformly distributed between 0 and a small angle $\theta_{\max }$. Periodic boundary conditions are used. Now a second grid is defined, having a grid constant 5-20 times smaller than that of the crystal positions. The time is increased step by step by an amount $\Delta t$. For each time step, we determine which crystals are visible at each fine grid point. So, the $2 \mathrm{D}$ ground plane is partitioned into regions in which only one crystal is visible. This region is surrounded by other regions. If the time is increased, the borders between the region under consideration and its neighbors will change; also, neighboring regions may disappear completely and new ones may arise. To calculate the new borders, for every region it is determined which are the visible crystals of its neighboring regions and these are put in a list. Then, for every point in the region, from this list extended with the crystal which is currently visible in that region, the crystal yielding the greatest height at this point is determined. After the whole fine grid has thus been scanned, all points are updated to their new values.

This procedure still allows for one of the neighboring regions to pop up in the middle of the region under consideration. Such events are most probable and most undesirable for the crystal tops. Therefore, every crystal is tagged whether its top has disappeared or does still persist at the surface. If a top tagged as disappeared reappears again, the region it is part of is cleared and filled with the crystals of its neighbors attaining the greatest heights. This algorithm still allows for regions popping up between other regions, if the new region does not contain tops and is part of the same crystal as one of the neighboring regions. This implies an overestimation of cone trunks, in contrast to mean-field theory, in which they are underestimated (see above). One is, therefore,

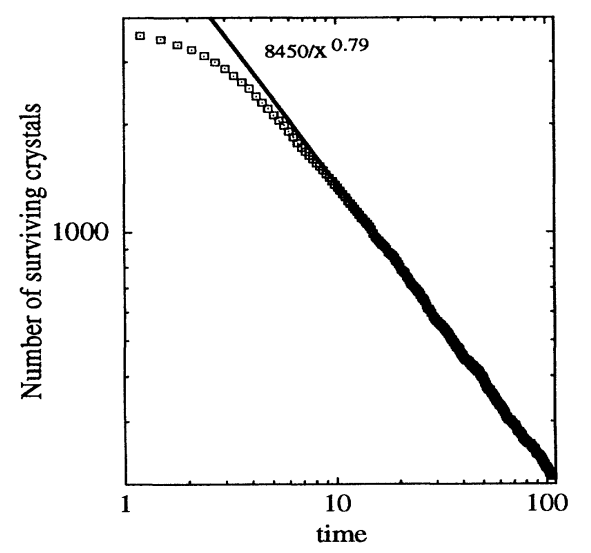

FIG. 5. The number of cone-shaped crystals surviving at the surface vs time on a log-log scale. Time is given in units of $d / v$ with $v$ the growth velocity and $d$ the distance between nearest-neighbor crystals. This simulation was performed for $60 \times 60$ crystals with a fine grid of $600 \times 600$ points and a time step $\Delta t=0.3$. The straight line has a slope 0.79 . This slope is twice the exponent $p$. confident that if the same exponent $p$ is found for both simulation and mean-field theory, it will be the correct one. The simulation is stopped when it becomes possible for boundary effects to show up.

Figure 5 shows the decrease of the number of surviving tops with time for cone-shaped crystals. The results are seen to fit a straight line with a slope 0.79 in a log-log plot very well. Averaging such slopes for ten runs with systems ranging from 3600 to 6400 cones, a value $p=$ $0.40 \pm 0.01$ is found for the domain size exponent (which is half the slope of the line shown in Fig. 5). The exponent remains unchanged for finer grid constants down to five times smaller than the cone grid. Also, for time steps smaller than 0.3 the same result is found. For cubes, the same exponent is found for the same parameter ranges as in the cone case. Again $p=0.40 \pm 0.01$, is found, based on averaging the results of ten simulations for systems up to 4900 cones.

Since the mean-field theory not only predicts the exponent, but also the distribution of surviving crystals as a function of the angle $\theta$ with the normal direction and time $t$, it is possible to check its validity in more detail. In fact, according to mean-field theory, the distribution should read

$$
P(\theta, t) \propto \theta \exp \left(-\theta^{5} t^{2}\right) .
$$

The factor $\theta$ before the exponent arises from the angular integration. ${ }^{8}$ If one now puts $x=\theta^{2} t^{4 / 5}$, the distribution becomes

$$
P(x) \propto t^{-4 / 5} \exp \left(-x^{5 / 2}\right) .
$$

For six independent cone simulations with $80 \times 80$ crystals, $t^{4 / 5} P(x)$ is measured over seven time segments. The curves collapse very well as can be seen from Fig. 6.

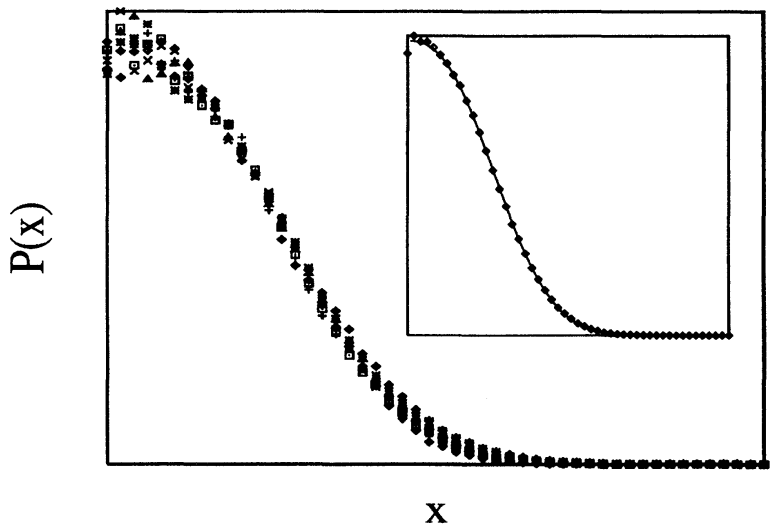

FIG. 6. Collapsed data for the probability distribution $P$ as a function of $x=\theta^{2} t^{4 / 5}$ in arbitrary units. Data obtained for a $80 \times 80$ cone system and a $500 \times 500$ fine grid. The seven curves are the results for seven different time segments ranging between $t=10.0$ and 246. Data are averaged values for six independent runs. The sum of the represented curves is shown in the inset. The drawn line in this inset shows the best fit of the data to a curve of the form $A \exp \left(-\alpha x^{q}\right)$ with $q=2.499$. 
Moreover, if the averaged curve is fitted to a function of the form $A \exp \left(-\alpha x^{q}\right)$, the optimum value found for $q$ is equal to $2.5 \pm 0.1$. Also for cubic crystals using a system of size $70 \times 70$, data-collapsing works well with an exponent $q=2.5 \pm 0.1$. It is thus clear that the mean-field theory yields the correct value for the growth exponent and that it also gives a good description of the detailed behavior of the model.

\section{ACKNOWLEDGMENTS}

I thank Hubert Knops for useful discussions. A. Zangwill kindly brought Ref. 9 to my attention. I am indebted to Peter Klok for giving me access to computer resources of the high-energy department of our university which were necessary for the completion of this work.
${ }^{1}$ W. van Enckevort, G. Janssen, W. Vollenberg, J. J. Schermer, and L. J. Giling, Diamond Relat. Mater. 2, 997 (1993).

${ }^{2}$ Chr. Wild, N. Herres, and P. Koidl, J. Appl. Phys. 68, 973 (1990).

${ }^{3}$ A. Van der Drift, Philips Res. Rep. 22, 267 (1967).

${ }^{4}$ A. J. Dammers and S. Radelaar, in Proceedings of the CP9O Europhysics Conference on Computational Physics, edited by A. Tenner (World Scientific, Singapore, 1991), pp. 310315; A. J. Dammers and S. Radelaar, Textures Microstruct. 757, 14 (1991).

${ }^{5}$ Chr. Wild, P. Koidl, W. Müller-Sebert, H. Walcher, R. Kohl, N. Herres, and R. Locher, Diamond Relat. Mater. 2, 158 (1993).

${ }^{6}$ R. P. U. Karunasiri, R. Bruinsma, and J. Rudnik, Phys. Rev. Lett. 62, 788 (1989); G. S. Bales and A. Zangwill, ibid. 63, 692 (1989).

${ }^{7}$ J. M. Thijssen, H. J. F. Knops, and A. J. Dammers, Phys. Rev. B 45, 8650 (1992).

${ }^{8}$ In Ref. 7 , a value 0.25 for $p$ was given. However, a trivial error was made in that paper by omitting an extra factor $\theta$ in the angular integration in Eq. (29). Putting this extra $\theta$ in Eqs. (29) and (30) leads to a $\theta^{5} t^{2}$ dependence of $\ln P(\theta, t)$ whence $p=0.4$.

${ }^{9}$ A. N. Kolmogorov, Dokl. Akad. Nauk SSSR 65, 681 (1949).

${ }^{10}$ P. Meakin, Phys. Rep. 235, 189 (1993).

${ }^{11}$ M. Kardar, G. Parisi, and Y.-C. Zhang, Phys. Rev. Lett. 56, 889 (1986).

12 C. Tang, S. Alexander, and R. Bruinsma, Phys. Rev. Lett. 64, 772 (1990). 